\title{
Controlling the Large-Scale Fabrication of Supraparticles
}

\author{
Alexander Plunkett, Catriona Eldridge, Gerold A. Schneider, and Berta Domènech*
}

Cite This: J. Phys. Chem. B 2020, 124, 11263-11272

Read Online

ABSTRACT: Controlling the nanoscale interactions of colloidal building blocks is a key step for the transition from single nanoparticles to tailor-made, architected morphologies and their further integration into functional materials. Solvent evaporationinduced self-assembly within emulsion droplets emerges as a fast, versatile, and low-cost approach to obtain spherical, complex structures, such as supraparticles. Nevertheless, some processstructure relationships able to describe the effects of emulsion conditions on the synthesis outcomes still remain to be understood. Here, we explore the effect of different physicochemical parameters of emulsion-templated self-assembly (ETSA) on supraparticles' formation. Supraparticle size, size dispersity, microporosity, and sample homogeneity are rationalized based

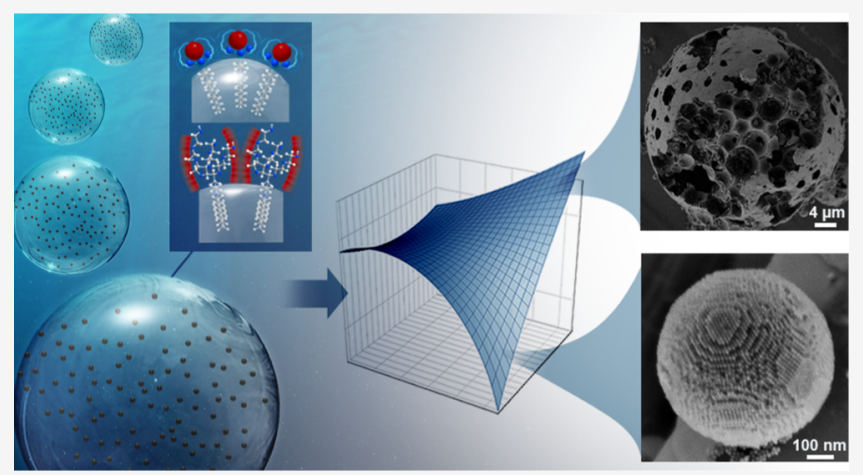
on the used surfactant formulation, stabilization mechanism, and viscosity of the emulsion. We further demonstrate the significance of the parameters found by optimizing a transferable, large-scale (gram-size) ETSA setup for the controlled synthesis of spherical supraparticles in a range of defined sizes (from $0.1-10 \mu \mathrm{m}$ ). Ultimately, our results provide new key synthetic parameters able to control the process, promoting the development of supraparticle-based, functional nanomaterials for a wide range of applications.

\section{INTRODUCTION}

Self-assembly has emerged as a key tool for arranging single, colloidally dispersed nanoparticles (NPs) into functional suprastructures. This assembly process relies on an interplay of several colloidal interactions between the involved nanobuilding blocks and the continuous phase in which they are dispersed. These forces, together with the kinetics of the process, will ultimately direct the features-in terms of, for example, shape or supercrystalline order-of the obtained condensed material and thus its final properties and applicability. ${ }^{1-4}$ Accordingly, the drive to obtain high-quality, NP complex structures for their further integration into novel functional materials and devices leads to the need of a good understanding and precise control of the synthetic parameters' effects. Among many developed experimental techniques, emulsion-templated self-assembly (ETSA) allows the preparation of well-defined spherical-shaped suprastructures, tunable in size and in NP arrangement. Through this technique, typically, NPs dispersed in oil droplets within an oil-in-water $(\mathrm{O} / \mathrm{W}$ ) emulsion (or other phase combinations i.e. $\mathrm{W} / \mathrm{O}, \mathrm{O} /$ $\mathrm{O}$ emulsions) arrange themselves into complex structures upon slow evaporation of the solvent carrier (oil phase). ${ }^{1,4-10}$ The obtained ETSA suprastructures consist of spherical particle agglomerates-with or without supercrystalline order-and are also referred in the literature as supraparticles (SPs), terminology which will be used in this work. ${ }^{4}$ Because SPs can remain in a dispersed form, they allow for an easy postprocessing, which is desired for their further applications in advanced optoelectronic devices, ${ }^{11-14}$ sensors, ${ }^{15}$ or tissue engineering $^{16}$ among others. Moreover, because the ETSA technique is applicable to a wide range of NPs of different materials, sizes, ${ }^{5}$ and shapes, ${ }^{6}$ the produced SPs appear as promising candidates for photonic materials ${ }^{17-20}$ or structural, hierarchical materials with outstanding mechanical properties. $^{21-27}$

The formation of emulsions is a nonspontaneous process, and the emulsified system is thermodynamically unstable. This makes an emulsion very prone to coalescence, sediment/ creaming, flocculation, and/or suffer from Ostwald ripening processes. During the emulsion process, though, the Gibbs free energy path for the emulsion formation can be tuned by the used surfactants and the viscosity of the continuous medium. ${ }^{28}$ Understanding the effect of these physicochemical conditions in the ETSA process therefore promises high control over the experimental outcomes.

Until now, several studies have been dedicated to control the kinetics of the self-assembly process and the NP's interactions within the emulsion droplets, , $^{4,7,10,16,29}$ or to obtain highly controllable and monodisperse SPs via non-

Received: August 10, 2020

Revised: November 2, 2020

Published: November 19, 2020 


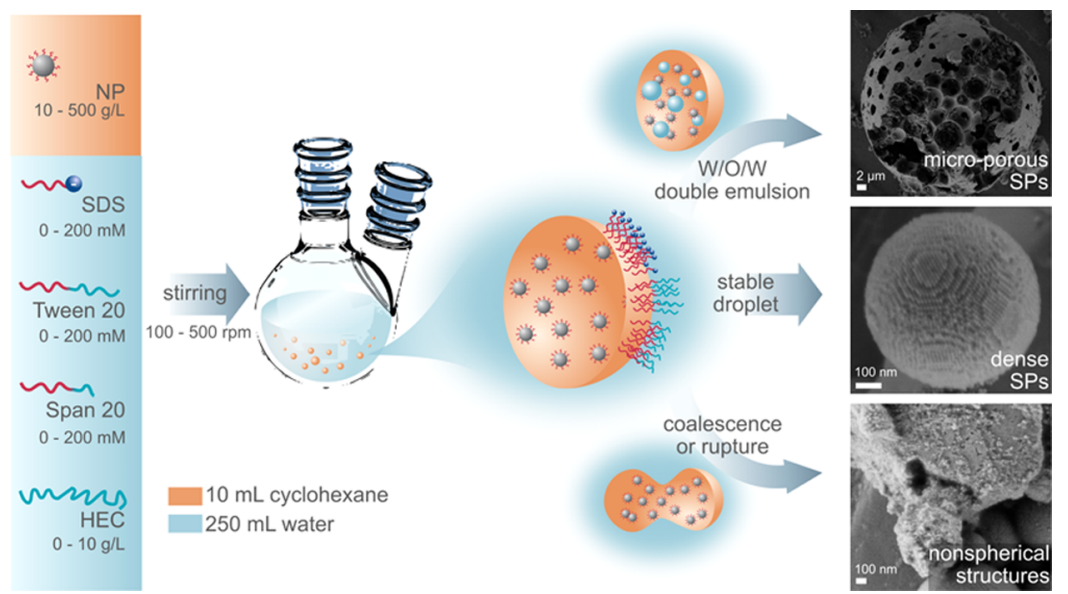

Figure 1. Schema of the used experimental setup with the input parameters and their ranges used to investigate the ETSA process. On the right, typical scanning electron microscopy (SEM) images of different ETSA outcomes, which range from microporous SPs, dense SPs, and nonspherical suprastructures. It is hypothesized that optimal emulsion droplet stability leads to the desired main product (supercrystalline dense SPs), while double emulsions (e.g. water-in-oil-in-water, W/O/W) and significant droplet formation and breakdown give undesired side-products (microporous SPs and/or nonspherical assemblies). Red and blue segments in the depicted surfactant molecules correspond to hydrophobic and hydrophilic moieties, respectively.

scalable setups, for example, microfluidics. ${ }^{4,6-8,30}$ Nevertheless, controlling the emulsion process itself while maintaining scalability is usually seldom addressed, and thus, the effects of some physicochemical conditions during ETSA are still not fully understood. ${ }^{31-35}$ This lack of control on the emulsion process leads to some effects for which the final outcome is unclear or not reported. An example of that is the usual appearance of undesired byproducts in the form of a variety of complex structures other than dense SPs. ${ }^{33,36}$ Because an emulsion is a dynamic system which may undergo rupture and coalescence of single droplets as well as formation of double emulsion droplets, the appearance of byproducts indicates a poor control on the emulsion properties. ${ }^{28} \mathrm{We}$ speculate that such transitions in the final state of SP formation are the main cause for the abovementioned byproducts (i.e. nonspherical suprastructures and microporous SPs, as shown in Figure 1), and therefore hypothesize that they can be controlled, or even avoided, by a better understanding of the emulsion process itself.

In this work, we examine the influence of some physicochemical, empirical parameters on the obtained ETSA outcomes taking as a base the fundamental concepts of emulsion stability. Specifically, we investigate the effect of different surfactant formulations, which tune the hydrophiliclipophilic balance (HLB), ${ }^{37}$ the stabilization mechanism (i.e. steric vs electrostatic), and the viscosity of the emulsion, on responses such as the obtained SP size, size dispersion, homogeneity (understood as a yield of the desired ETSA outcome), and fraction of microporous SPs. For a comprehensive understanding of these influences, we apply the concept of Design of Experiments (DoE). ${ }^{38}$ This allows us to model the effect of the different experimental input parameters on the synthesis outcomes with standard statistical techniques. $^{38,39}$ By this means, we establish a systematic, multivariate exploration of parameter effects and resolve their cross-dependencies, to reveal and understand some processstructure relationships. ${ }^{38-40}$ Based on the obtained DoE results, we further optimize the emulsion formulation for the synthesis of desired SPs (dense SPs in a specific size range) in a gram-scale setup, demonstrating how the gained under- standing can maximize the potential of this versatile, scalable, and cost-efficient approach.

\section{METHODS}

Materials. Cyclohexane (CHEMSOLUTE, $\geq 99.8 \%$ ), hydroxyethyl cellulose (HEC, Sigma-Aldrich), polyoxyethylene (20) sorbitan monolaurate (Tween 20, AppliChem, $\geq$ $100 \%)$, sodium dodecyl sulfate (SDS, Chemsolute, $\geq 98.0 \%$ ), and sorbitan monolaurate (Span 20, Merck, synthesis grade) were used without further purification. Oleic acid-stabilized $\mathrm{Fe}_{3} \mathrm{O}_{4} \mathrm{NPs}$ (CAN GmbH, Germany, $15.2 \pm 1.2 \mathrm{~nm}, 11.5 \mathrm{wt}$ $\%$ organic content, in toluene) were dried in vacuum and redispersed in cyclohexane before use.

Supraparticle Synthesis. A solution of 0.00165-200 mmol L ${ }^{-1}$ Tween 20, 0.00165-200 $\mathrm{mmol} \mathrm{L}^{-1}$ Span 20, $2.23-$ $200 \mathrm{mmol} \mathrm{L}^{-1} \mathrm{SDS}$, and $0.0100-10.0 \mathrm{~g} \mathrm{~L}^{-1} \mathrm{HEC}$ in $150 \mathrm{~mL}$ water was prepared in a $250 \mathrm{~mL}$ three-neck round flask via mild stirring at $50{ }^{\circ} \mathrm{C}$ until HEC was dissolved. After allowing to cool to room temperature, a suspension of $10-500 \mathrm{~g} \mathrm{~L}^{-1}$ $\mathrm{NP}$ in $10 \mathrm{~mL}$ cyclohexane was added to the aqueous solution. The flask was sealed, and the two phases were emulsified by vigorously stirring at $100-500 \mathrm{rpm}$ using a $68 \times 24 \times 3 \mathrm{~mm}$ poly(tetrafluoroethylene) stirrer shaft attached to a mechanical stirrer for $2 \mathrm{~h}$. Subsequently, the flask was opened to allow evaporation of the solvent, and the stirring was reduced to 60 rpm in order to prevent sedimentation and creaming. After 20 $\mathrm{h}$, the obtained suprastructures were separated from remaining free NPs in the surfactant solution by centrifugation at $5000 \mathrm{~g}$ for $20 \mathrm{~min}$, decanting the supernatant, and redispersion in 0.2 mmol L $\mathrm{L}^{-1}$ of Tween 20 aqueous solution. The purification step was repeated three times.

Sample Characterization. Scanning electron (SE) micrographs were acquired with a Zeiss Supra VP55 (Zeiss, Germany) at $1.5 \mathrm{kV}$, with a $10 \mu \mathrm{m}$ aperture size, in a high vacuum mode and using the Everhard-Thornley detector. Specimens were applied onto a SEM sample holder by dropcasting a dilute suspension of the ETSA outcomes in ethanol. Dynamic light scattering (DLS) measurements were carried out on a Malvern Mastersizer 3000 system attached with a Hydro EV dispersion unit using a $4 \mathrm{~mW}, 632.8 \mathrm{~nm}$ 

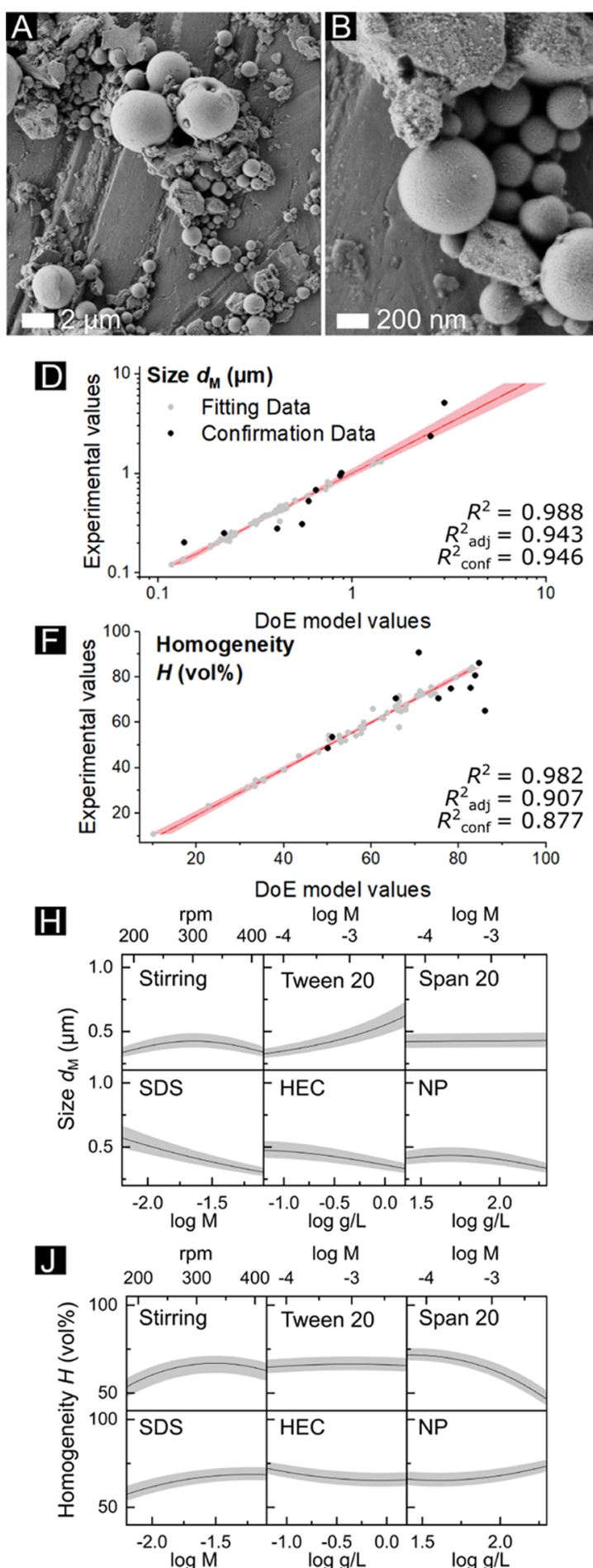

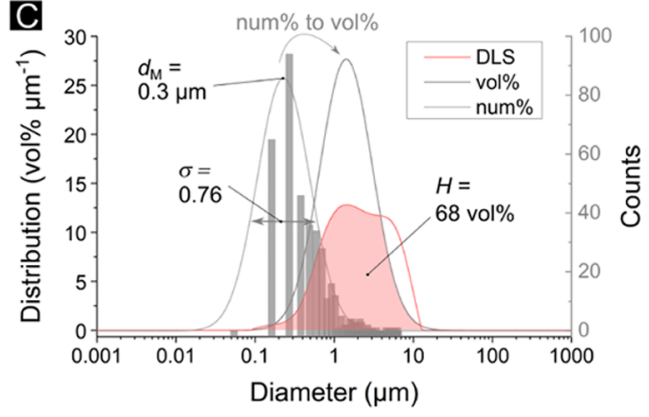

目

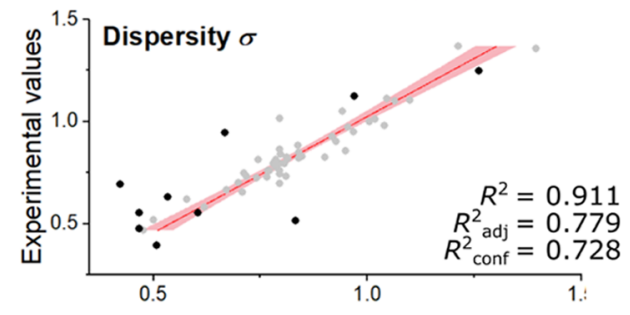

G

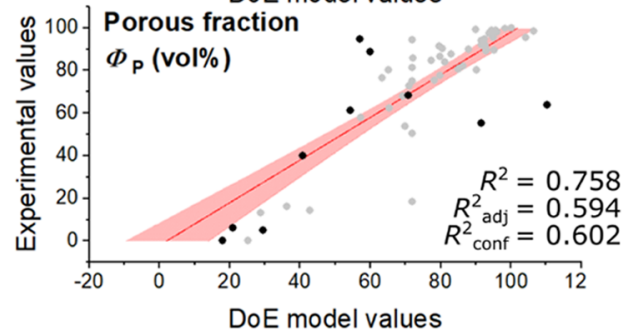

1

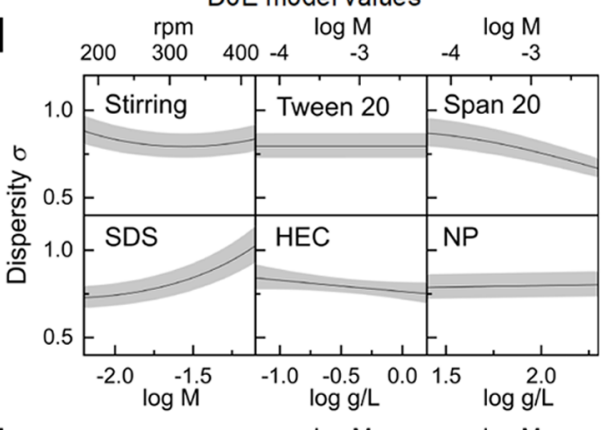

$\mathrm{K}$

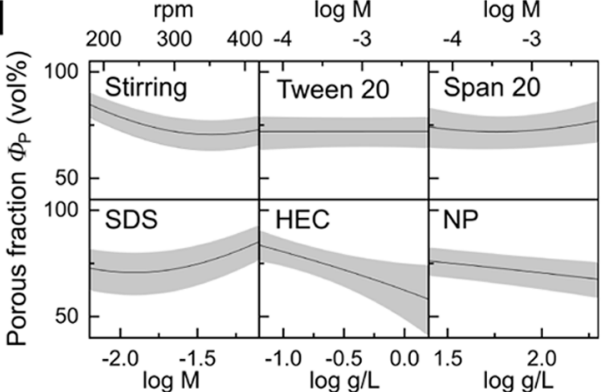

Figure 2. (A,B) SEM images of an example batch obtained only with SDS as the surfactant, showing the inhomogeneity of the obtained suprastructures, i.e. significant side-product appearance. (C) Comparison of the determined size distributions via manual SEM image evaluation and DLS for the sample shown in A and B. The similarity of the two distributions (i.e. the overlap integral) was used as a measure to quantify the homogeneity, H. (D-G) Model prediction ability by comparison of the DoE model prediction values versus the experimental values for SP size, $d_{\mathrm{M}}$, dispersity, $\sigma$, homogeneity, $H$, and fraction of porous SPs, $\Phi_{\mathrm{P}}$. The coefficient of determination, $R^{2}$, and the adjusted coefficient of determination, $R_{\mathrm{adj}}{ }^{2}$, are only related to the fit data, whereas the coefficient of determination, $R_{\text {conf }}^{2}$, also refers to the confirmation data. (H-K) Univariate slices on the response surface for each model at the parameter settings of the center point. Note that univariate representations do not display cross-dependencies of the input factors. More information can be found in the Supporting Information, Section 4.

$\mathrm{He}-\mathrm{Ne}$ laser and $10 \mathrm{~mW}, 470 \mathrm{~nm}$ light-emitting diode illumination. From SEM images, 300 (dense and porous) SP diameters per sample were evaluated, and the results fitted to a log-normal distribution to extract the SP size, $d_{\mathrm{M}}$, and the size dispersity, $\sigma$ (i.e. standard deviation, extracted from a lognormal distribution fit). The fraction of microporous SPs, $\Phi_{\mathrm{P}}$, was obtained by visually identifying and manually classifying 
the amount of porous versus dense counted SP diameters and calculating the corresponding volume fraction.

DoE Model Building. The experimental design, calculation of empirical models, and their evaluation were carried out using Design-Expert 12 (Stat-Ease, Inc., Minneapolis, MN, USA). A resolution VI 6 factor factorial $2^{k-1}$ design augmented by star points with distance $\alpha=2^{5 / 4}$ to a Central Composite Design covering 48 experiments, including four replicates of the center point, was applied. The parameter settings are listed in Table S1. Because a linear scale did not allow to observe significant effects, the concentration parameters (i.e. concentrations of Tween 20, Span 20, SDS, HEC, and NPs) were applied in a logarithmic scale.

Models in the form of polynomial expressions representing the experimental output as a function of input variables are constructed from the most optimally statistically significant polynomial terms and fitted to the design data. The model selection was done algorithmically by optimizing the adjusted coefficient of determination, $R_{\mathrm{adj}}{ }^{2}$, using a critical $p$-value of 0.1 . The obtained model terms and their statistical significance are listed in Tables S2-S5. Box-Cox power transformation $^{38,41}$ was employed to stabilize variance in case of heteroscedasticity and noted with the transformation factor, $\lambda$.

Models were accepted after validating with 10 additional confirmation experiments on the basis of three criteria: (i) difference between the adjusted coefficient of determination, $R_{\mathrm{adj}}^{2}$, and the coefficient of determination, $R^{2}$, being lower than 0.2 , (ii) the confirmation set coefficient of determination, $R_{\text {conf }}^{2}$, approaching the fitting set, $R^{2}$, and (iii) each model term having a high degree of statistical significance (a $p$-value of approximately 0.1 or lower).

\section{RESULTS AND DISCUSSION}

Multivariate Parameter Analysis. NP complex structures were synthesized via ETSA, as described in the Methods section. In brief, NPs were dispersed in cyclohexane and added to an aqueous solution of surfactants and thickener followed by emulsification via intense stirring in a $250 \mathrm{~mL}$ round-bottomed flask equipped with a mechanical stirrer (Figure 1). Further mild stirring allowed the cyclohexane to evaporate during a period of $20 \mathrm{~h}$. After evaporation of the NPs' solvent, the obtained complex structures appeared in a mixture of morphologies, ranging from microporous SPs, dense SPs (i.e. without micropores, henceforth named as SPs), and nonspherical suprastructures ${ }^{33,36}$ (high-resolution micrographs of some examples can be found in the Supporting Information, Section 2).

The emulsion's physicochemical conditions were modified by adjusting the concentration of SDS, polyoxyethylene (20) sorbitan monolaurate (Tween 20), and sorbitan monolaurate (Span 20) as ionic $(\mathrm{HLB}=40)$, hydrophilic nonionic $(\mathrm{HLB}=$ 16.7), and lipophilic nonionic surfactants (HLB = 8.6), respectively. ${ }^{37}$ Moreover, we modified the applied shear and the viscosity of the aqueous phase, via stirring and the addition of HEC as a thickener (Figure 1). The starting NP concentration varied, to adjust the initial droplet properties.

A total of 48 experiments were performed according to a response surface design. As responses, the most frequent sphere diameter (defined as size, $d_{\mathrm{M}}$ ), size dispersity $(\sigma)$, sample homogeneity $(H)$, and fraction of microporous SPs $\left(\Phi_{\mathrm{P}}\right)$ were obtained from SEM and DLS analyses. Specifically, the $d_{\mathrm{M}}, \sigma$, and $\Phi_{\mathrm{P}}$ were obtained from analyzing $\mathrm{SE}$ micrographs, as described in the Methods section. As a figure of merit for the homogeneity, we defined the overlapping area, $H$, of the normalized volume distribution from SEM measurements with the DLS distribution (further information in the Supporting Information, Section 3). This is exemplified in Figure 2A-C. When only SDS is used as a stabilizing agent, ${ }^{9,10,36}$ the obtained suprastructures are highly inhomogeneous, ranging from spherical SPs to nonspherical NP agglomerates. The obtained diameter distribution curves of these samples (Figure 2C) show in fact a significant mismatch between the measurement methods, DLS and SEM. Although in the manual SEM-based size measurement, only spherical complex structures were considered, DLS measures all suprastructures regardless of their shape, including the nonspherical ones. Therefore, improvement of sample homogeneity would lead to an increase of the overlapping integral. It has to be highlighted that the overlap integral does not represent the absolute value of the SP content within the samples.

The measured responses (size, size dispersity, homogeneity, and porous SP fraction) of the conducted experiments were used to obtain DoE models using the software Design-Expert (Stat-Ease, Inc.). By comparing the experimental values of the measured responses (see Fitting Data in Figure 2D-G) versus the model-calculated responses for each parameter setting, we see a good agreement between both (exemplified by the $R^{2}$ and the $R_{\mathrm{adj}}^{2}$-obtained values). This indicates that each developed model is able to properly represent the obtained experimental outcome. For further verification, additionally, the models were tested with confirmation tests by conducting 10 additional experiments with parameter settings different from the ones used in the design experiments (see Confirmation Data in Figure 2D-G). Comparing the confirmation experimental outcomes with the prediction provided by DoE models for each response (Figure 2D-G, detailed DoE model terms are presented in the Supporting Information, Section 4) allowed us to confirm that the developed models have a good power of predictability for the used system (as exemplified by the obtained $R_{\text {conf }}^{2}$ ) and thus can be used to further rationalize the ETSA process based on the studied parameters.

All models, for all investigated responses, exhibit dependencies on all six input parameters (stirring $\mathrm{rpm}$ and concentrations of Tween 20, Span 20, SDS, HEC, and NPs), as well as complex interactions between them (Tables S2-S5). Hence, the depicted univariate "slices" in the response surface (Figure 2G-I) give an overall impression on each parameter influence, however, the detailed relationships change depending on all the other parameter settings. The impact of each model term on the experimental output, and thus possible parameter interactions, is represented by the coded coefficient values provided in Figure S7.

In summary, it is found that mainly the surfactants Tween 20 and SDS determine the SP size, while the applied shear is less significant (Figure $2 \mathrm{H}$ ), pointing out toward a thermodynamic control of the system rather than a kinetic one (under the studied conditions). Increasing the amount of SDS generally leads to smaller SP sizes, while an increase of Tween 20 provokes the opposite effect. This can be explained based on the hydrophilic and lipophilic moieties present in the used surfactants, represented by their HLB values. ${ }^{37}$ The HLB value allows relating the effect of the different intermolecular forces acting between surfactant molecules and surfaces (repulsive hydrophilic between headgroups, attractive hydro- 


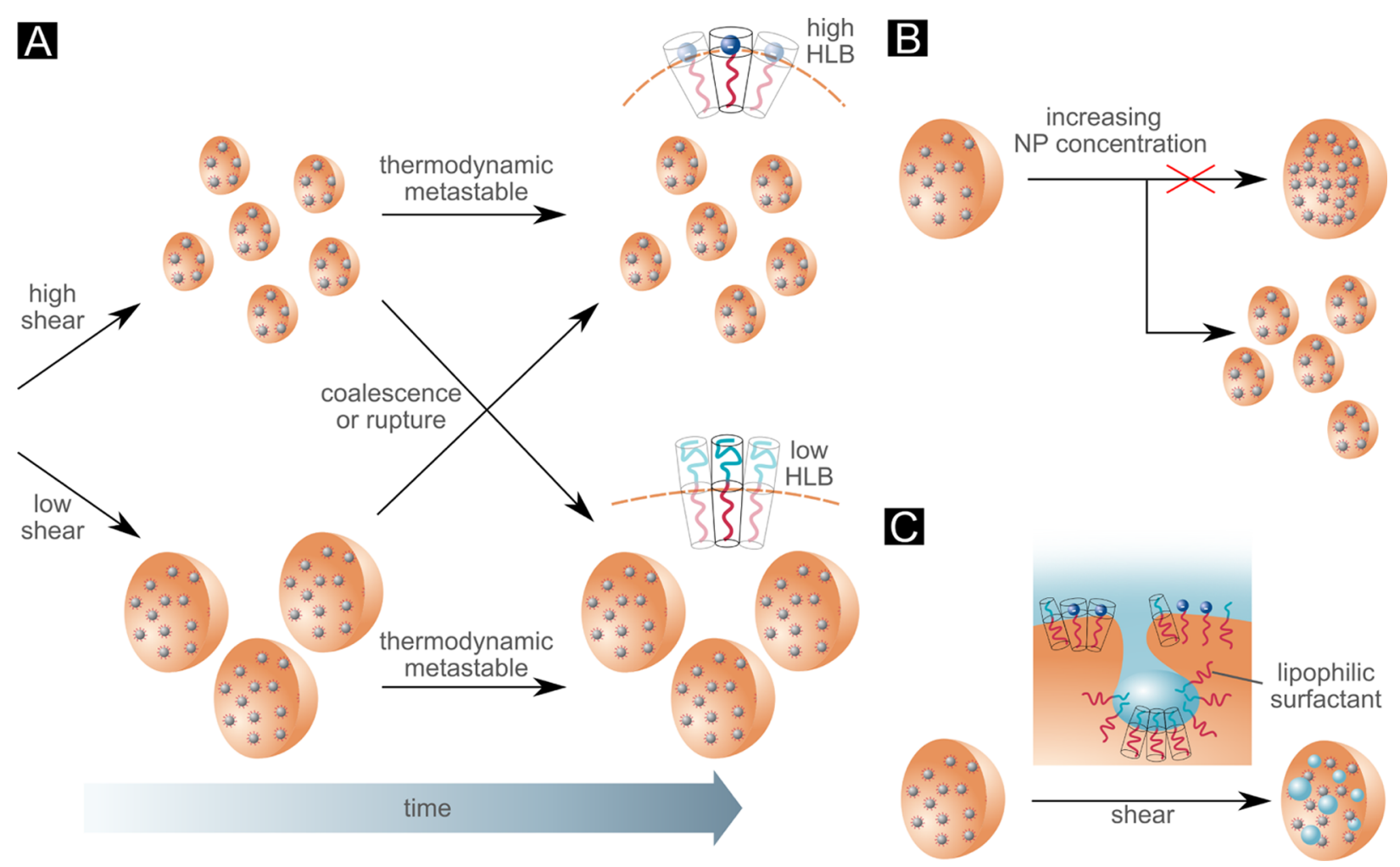

Figure 3. Proposed mechanism of some selected physicochemical effects on the ETSA outcomes. (A) Although the applied shear can adjust the initial droplet size distribution under certain emulsion conditions, the system moves toward a thermodynamic metastable state determined by the critical packing radius of the surfactant blend. (B) Higher starting NP concentrations do not lead to bigger SPs under the same process conditions. Instead, the formation of smaller droplets is favored leading to smaller SPs. (C) Combining ionic and lipophilic surfactants favors the formation of $\mathrm{W} / \mathrm{O} / \mathrm{W}$ double emulsions ultimately leading to microporous SPs. The ionic surfactant does not sufficiently prevent the entering of small water droplets into the oil phase. Once formed, the interior water droplet is then stabilized by the lipophilic surfactant.

phobic at the hydrocarbon-water interface, and repulsive steric forces between aliphatic chains) to the geometry of the surfactants within the emulsion and ultimately to the droplet's size. High HLB surfactants, like SDS (HLB = 40), feature a large hydrophilic head group compared to their hydrophobic tail. This allows them to pack as "sharp cones" into higher droplet curvatures than steric surfactants, such as Tween 20 $($ HLB $=16.7) .{ }^{42}$ Therefore, when high HLB surfactants are used, smaller droplet diameters are stabilized and consequently smaller SPs are obtained. This is schematically summarized in Figure 3A.

Surprisingly, higher starting NP's concentrations generally do not lead to bigger SPs. Intuitively, one would assume that a higher NP load, under the same process conditions, would lead to the integration of a higher number of NPs within the droplets, and thus to larger SPs, as shown in Figure 3B. Contrarily, the results indicate that a higher NP load favors the formation of smaller oil droplets. Strong interactions of the NP concentration parameter with all the surfactant terms (Figure S7A) indicate that the increase in the number of oilcontained NPs is able to change the properties of the oil phase, in a way that the system no longer behaves like a pure cyclohexane-in-water emulsion.

The size dispersity is also strongly influenced by the choice of surfactants and thickener, yet mainly by Span 20 and SDS (Figure 2I); while the applied shear (stirring) has no effect on the size nor the size dispersity. Again pointing out toward a thermodynamic control of the emulsion by the physicochemical conditions, it is to say, by the specific combination of surfactants and thickener. Nevertheless, it is important to note that the sample homogeneity shows a strong dependency on the applied stirring (Figure 2J) yet with the local maxima with strong cross-dependencies on the surfactants used (Figure $\mathrm{S} 7 \mathrm{C})$. For a certain surfactant formulation, when the intensity of applied shearing forms a droplet size distribution which is thermodynamically metastable, a significant reduction of droplet coalescence and rupture can be expected, as depicted in Figure 3A. Furthermore, the obtained homogeneity is generally higher when using mixtures of surfactants instead of single-surfactant emulsions. This again relates to the stability of the emulsion because the right combination of surfactants-especially when combining ionic and nonionic surfactants-can reduce coalescence as a result of several effects such as high Gibbs elasticity, high surface viscosity, and hindered diffusion of surfactant molecules from the interface's film. ${ }^{28}$ These findings support the proposed mechanism in Figure 1 and allow reducing the amount of byproducts by diminishing the frequency of droplet reformation.

The fraction of porous SPs shows a positive dependency on the concentration of SDS and Span 20 and a negative effect when increasing the concentration of HEC (Figures $2 \mathrm{~K}$ and $\mathrm{S} 7 \mathrm{D})$. Considering the proposed mechanism of $\mathrm{W} / \mathrm{O} / \mathrm{W}$ double emulsions leading to microporous SPs in Figure 1, it is in fact expected that ionic surfactants allow small water droplets to enter a pre-existing oil droplet more easily than sterically stabilizing surfactants. Once formed, the small interior water droplets are then stabilized by the hydrophobic, low HLB surfactants, such as Span 20, as illustrated in Figure 

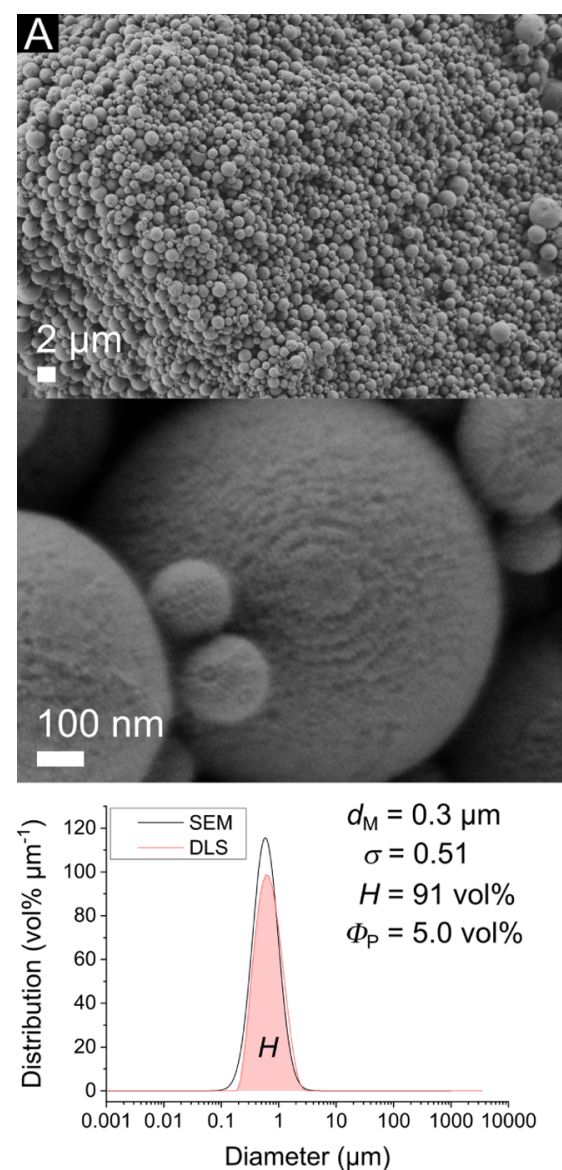
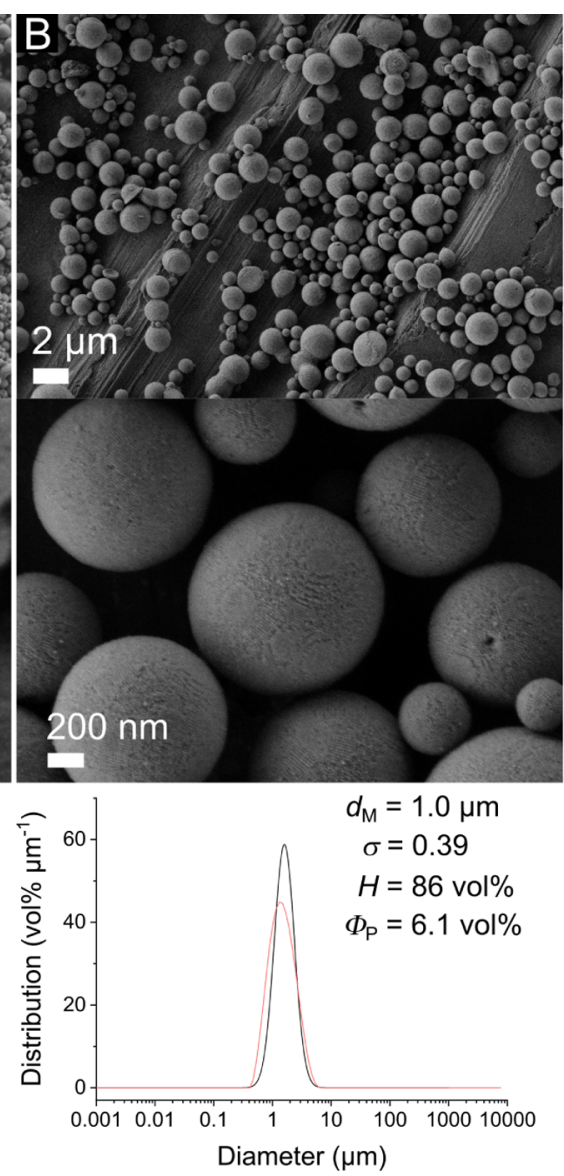

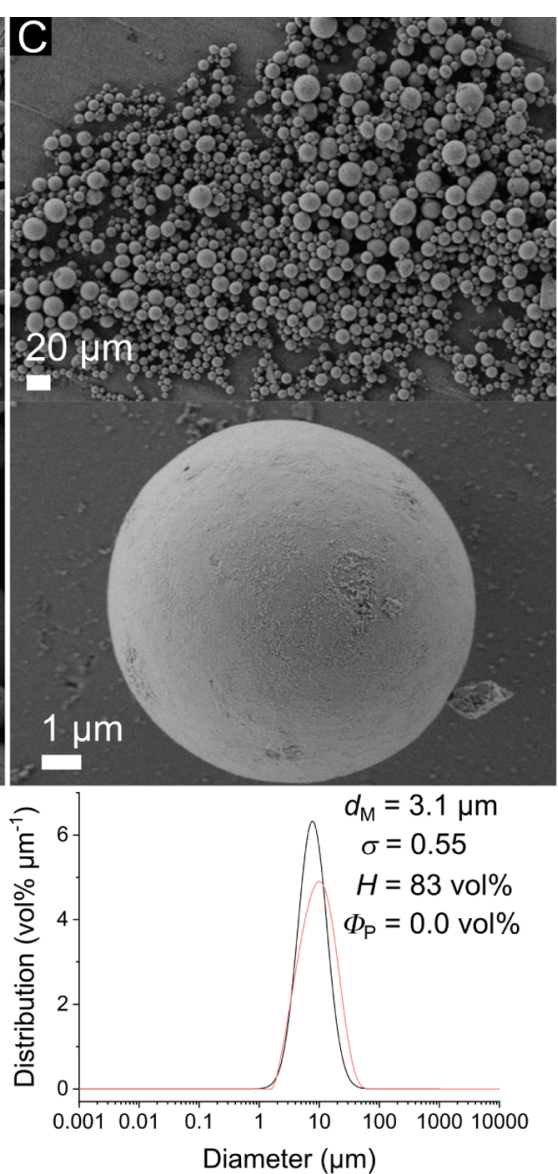

Figure 4. Optimized samples with a most frequent size $0.3 \mu \mathrm{m}$ (A), $1.0 \mu \mathrm{m}$ (B), and $3.1 \mu \mathrm{m}$ (C). The homogeneity, $H$, of the samples is indicated by the similarity of size distribution obtained via SEM and DLS. Please note that the shifting of the most frequent size, $d_{\mathrm{M}}$, in the spectra toward higher values is because of conversion from number distribution to volume distribution. More information is given in Figure S9.

3C. Furthermore, a general decrease in the porous SP fraction is observed when simultaneously increasing homogeneity and decreasing dispersity, indicating a strong correlation between these responses (Figure S8). This is most likely due to the fact that microporous SPs are more fragile and often led to small fragments within the samples (Figure S4), which is detected as mismatch between SEM and DLS measurements. Moreover, because for the same NP content, SPs containing voids naturally have a bigger diameter than micropore-free SPs; this directly reflects on the relation between microporosity and size dispersity.

Nevertheless, it has to be noted that the porous fraction model (outcomes summarized in Figure 2G,K) has a lower statistical significance and hence lower prediction power (compare $R^{2}, R_{\mathrm{adj}}^{2}$, and $R_{\text {conf }}^{2}$ in Figure $2 \mathrm{D}-\mathrm{G}$ ) than the rest of developed models. Limitations such as the incomplete sphere porosity assessment (e.g. micropores were not always superficially visible) very likely hindered the obtaining of a highly statistically significant model. Thus, even though the porous model allows us to understand and rationalize the parameter influences, its low prediction power hinders a further optimization of this feature.

All in all, the described effects and the cross-dependencies indicate how the system outcomes strongly rely on specific physicochemical adjustments (i.e. emulsion droplet stability). Therefore, adjusting the starting composition of the emulsion based on the knowledge provided by the parametric analysis should allow to obtain an ETSA-specific desired outcome.
Optimized Transferable, Large-Scale ETSA. Based on the developed models and the gained understanding of the process, a further adjustment of the experimental physicochemical conditions was used to obtain targeted SP sizes and minimize the side-product formation with a simple setup. Hence, optimal self-assembly conditions were found by numerically predicting compromise solutions for the obtained models and to produce dense SPs with three different average sphere sizes with minimum dispersity and maximum homogeneity (detailed experimental settings shown in the Supporting Information, Section 5). Because of the low statistical significance and compensability of the abovementioned porous fraction model, this model was not considered for the optimization.

As can be observed in Figure 4, the optimized samples show a significant improvement (compared to the ones presented in Figure 2 and Supporting Information Section 2) in terms of dispersity $(\sigma \leq 0.55)$ and sample homogeneity ( $\geq 83 \mathrm{vol} \%)$, as shown by the good match between the DLS spectra and the manually counted SEM size distribution (details are given in the bottom part of Figure 4). This demonstrates that this quantification method is sufficient to find trends for improvement within the DoE models. It is also important to note that, although the size dispersity of these optimized samples is significantly improved, the values here reported represent the limit of the applied system, as predicted by the DoE model. The SP sizes are yet log-normal distributed as a result of the formation of the emulsion droplets by shear 
A

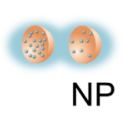
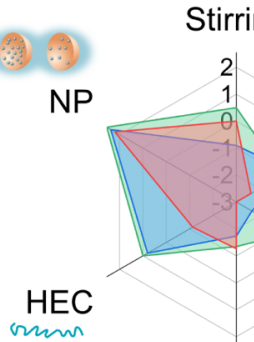

Stirring

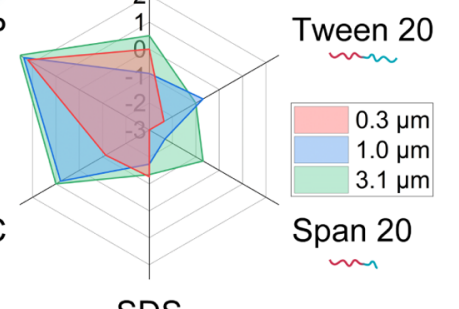

SDS
B

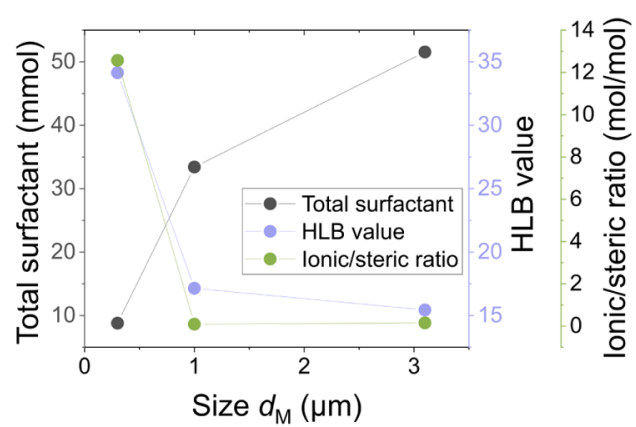

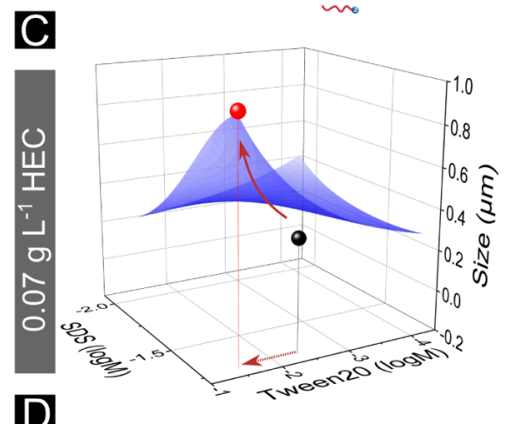
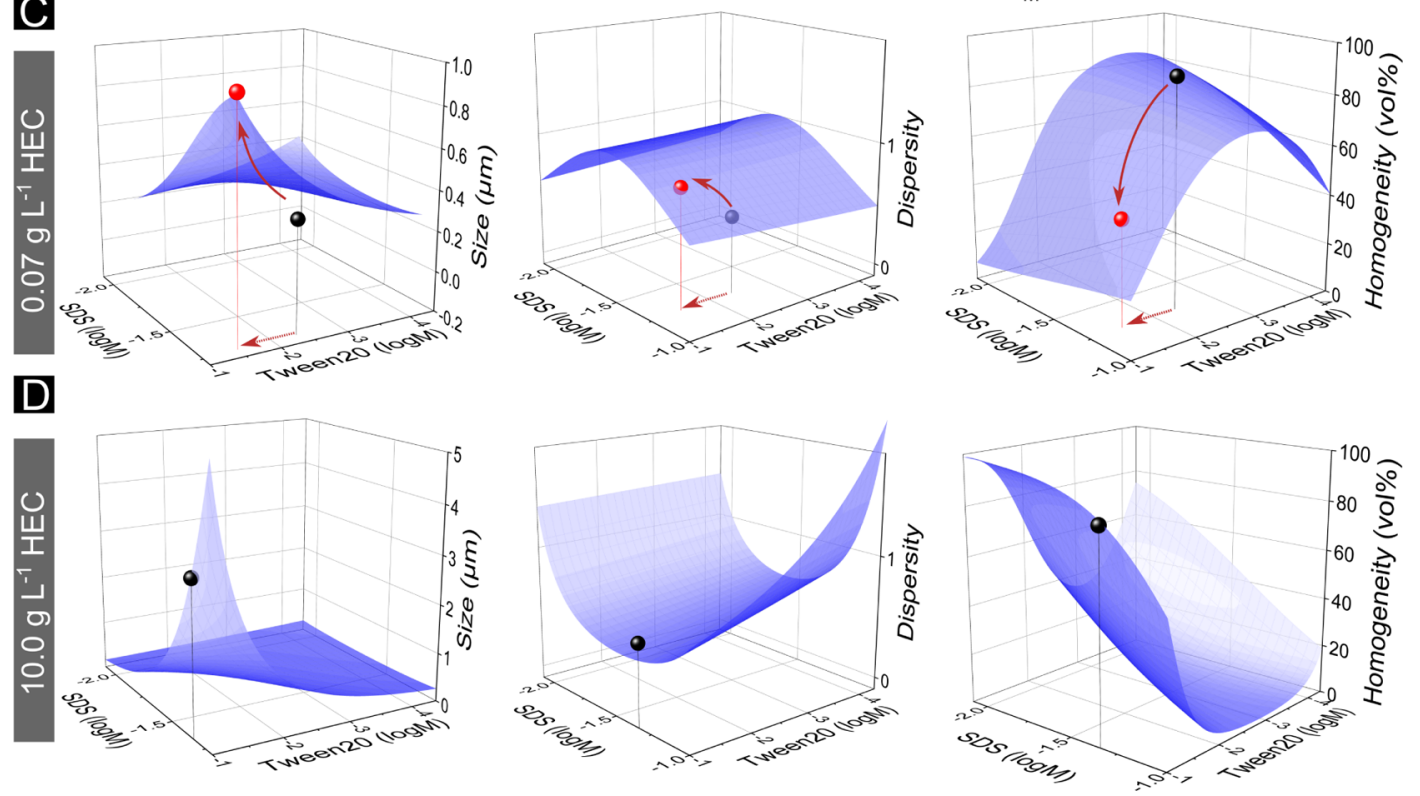

Figure 5. (A) Parameter settings for three optimized SP sizes, $0.3,1.0$, and $3.1 \mu \mathrm{m}$. The dimensions are given in coded values $[-\alpha$ to $+\alpha]$ for the sake of simplicity. (B) Required total surfactant, blend HLB value, and ratio of ionic and steric surfactants for the optimized samples. Response surface plots of size, dispersity, and homogeneity at low (C) and high viscosity (D) conditions (determined by HEC concentrations) as a function of SDS and Tween 20 concentrations. Black spheres represent optimized sample conditions for 0.3 and $3.1 \mu \mathrm{m}$. The red sphere in (C) indicates how the system would behave if, with the aim of increasing the size at a low HEC concentration, the concentration of surfactants is increased.

forces (i.e. stirring). ${ }^{28}$ Nevertheless, the gained understanding for optimized physicochemical conditions presented herein can also be applied in techniques with a specific spherical spatial constraint, like membrane emulsification ${ }^{43}$ or in microfluidics setups.

Even though the model for $\Phi_{\mathrm{P}}$ was not considered in the optimization step, the optimized samples also exhibit a significant reduction of the porous SP fraction compared to the previously designed experiments. Therefore, the optimization of sample homogeneity and dispersity per se includes a reduction of the microporous SP content. This is in agreement with the results found in the multivariate parameter analysis, and related, as discussed before, to the crossdependency of this parameter with both, homogeneity and size dispersity.

Insights on the Optimized ETSA Process. A closer look at the determined parameter settings used for obtaining the optimized samples (Figure 5A, and Table S6) reveals an optimal stirring around the center point (i.e. $300 \mathrm{rpm}$ ) and an optimal starting NP concentration at higher values (160-455 $\left.\mathrm{g} \mathrm{L}^{-1}\right)$. We hypothesize that a high starting NP concentration leads to an earlier onset of self-assembly and therefore a smaller timeframe for side-products to form. Interestingly, the optimal surfactant and thickener formulation significantly differ for the different SP sizes. As expected, based on the previously discussed parametric study analysis, the required blend HLB value decreased with the increasing SP size (Figure 5B), again indicating that the resulting critical packing radius $^{42}$ plays a major role in determining the final SP size. Comparing the sum of Tween 20, Span 20, and SDS, a total surfactant amount of $8.8,33.4$, and $51.5 \mathrm{mmol}$ was required to obtain spheres of sizes $0.3,1.0$, and $3.1 \mu \mathrm{m}$, respectively (Figure 5B). Thus, larger SPs required an increased amount of the total surfactant despite having a smaller total surface area. Increasing surfactant concentrations in the bulk of the aqueous phase likely causes an increase of surfactant molecules bound to the surface of the oil droplet, decreasing the surface tension and hence increasing their stability. ${ }^{28}$ Additionally, the required ratio of ionic and steric surfactants decreased with increasing SP size (Figure 5B), which could again be attributed to the stronger reduction of surface tension per molecule for Tween 20 compared to SDS. ${ }^{44}$ Therefore, it can be stated that bigger SPs require a stronger stabilization compared to smaller ones, which relates to the higher creaming rate of larger emulsion droplets which puts them at risk of coalescence. ${ }^{28}$

The response surfaces at the parameter settings used for 0.3 and $3.1 \mu \mathrm{m}$ spheres with low and high HEC concentrations 
are presented in Figure 5C,D, respectively. From these, it can be noted that the viscosity of the aqueous media (determined by the concentration of HEC) played an important role in obtaining larger SPs with a narrow size distribution (Figure 5C). As concluded from parametric analysis, increasing the amount of Tween 20 would be a trivial way to increase the SP size, for example, starting from the parameter coordinates used for $0.3 \mu \mathrm{m}$ spheres (black sphere in Figure 5C) at a low viscosity. However, as indicated by the red sphere in Figure $5 \mathrm{C}$, this would also significantly increase dispersity and reduce homogeneity of the sample. Thus, to obtain larger SPs with high homogeneity and low dispersity, the viscosity has to be also increased (black sphere in Figure 5D). In fact, the positive effect of viscosity is well known for basic emulsions because reducing turbulence leads to larger droplets with a narrow size distribution and decreases their creaming rate. ${ }^{28}$ Here, it is now shown that this effect also applies to ETSA NP systems. Interestingly, a simple increase of the viscosity (without increasing the amount of the surfactant) does not lead to improved sample quality for smaller SPs. One explanation could be that high HEC to surfactant ratios could lead to HEC being the main stabilizing agent of the emulsion droplets and/or to the complexation of HEC with the surfactants, ${ }^{45}$ leading to an insufficiently stabilized emulsion system.

Based on the gained understanding of the system, and following the optimized formulation for obtaining the SPs of $1.0 \mu \mathrm{m}$ in size, a batch of $10 \mathrm{~g}$ of optimized SPs was obtained (Figure S10). This demonstrates the applicability of this approach for the production of larger batches with a controlled outcome.

It is also worth noting that formulations leading to homogeneous samples with a predominant fraction of microporous SPs could also be obtained. The formation of micropores in such suprastructures is itself a topic of great interest. In the reported literature, hierarchical porosity is usually achieved by co-assembling sacrificial polymer particles within the spherical SPs. ${ }^{16,46}$ Nevertheless, for a single particle-type ETSA process, micropore formation is usually neither studied nor controlled. In this work, based on multivariate parameter analysis, the surfactant composition was purposefully adjusted to high ratios of SDS (89-95 mol $\%)$ and moderate amounts of Span 20 (3-11 mol \%). These compositions allowed us to obtain samples with high microporous SP fractions (see the Supporting Information, Section 6). This combination of surfactants-high concentrations of ionic surfactants combined with a low HLB, lipophilic surfactant-promotes the formation of $\mathrm{W} / \mathrm{O} / \mathrm{W}$ emulsion droplets, which ultimately results in microporous SPs, confirming the mechanism proposed in Figure 1 and in Figure 3C. In fact, this is in agreement with a previous observation for paraffin-in-water emulsions, ${ }^{47}$ although, to the best of the authors knowledge, it is for the first time reported for the ETSA process. Additionally, these findings deliver a strong hint on how to tune the pore size in the microporous SPs. Similar to the control of the SP size by adjusting the blend HLB of the hydrophilic surfactants, adjusting the HLB value of the lipophilic surfactant would determine the size of the interior water droplets and ultimately the micropore size.

\section{CONCLUSIONS}

ETSA allows to obtain NP suprastructures in a fast and reproducible way. Herein, we explored the effect of different experimental physicochemical parameters during the ETSA of spherical NPs to obtain targeted SPs with defined features. The significance of parameters such as shear, concentration of surfactants and NPs, type of surfactants, and viscosity of the aqueous phase has been evaluated based on the obtained SP size, size distribution, and the formation of side-products (nonspherical structures or microporous SPs). Nonlinear cross-dependencies between the different parameters emerged, which makes the system challenging to solve by usual monothetic experimental approaches. Statistical DoE allowed to resolve some of these cross-dependent effects and to understand process-structure relationships based on emulsion thermodynamic principles, allowing us to establish some new key experimental parameters for controlling the ETSA outcomes. We found that blends of ionic and nonionic surfactants lead to improved sample homogeneity because of combined electrostatic and steric barriers against coalescence. The blend HLB value of the surfactant formulations appeared as a key parameter for controlling the critical packing radius of the emulsion droplets and thus the final SP mean size. It also emerged that to overcome the higher risk of creaming and coalescence for emulsions with big droplet size, steric stabilization and an increased amount of total surfactants and thickener are required. This allowed us to obtain larger SPs by stronger stabilization via reduction of the interfacial tension in a viscous aqueous phase. Finally, this gained knowledge allowed us to obtain homogeneous gram-scale batches with controlled SP sizes from $0.1-10 \mu \mathrm{m}$. Moreover, the obtained results also give important hints on how the SP's microporosity can be tuned by adjusting the surfactant mixtures.

The study hereby presented therefore complements the understanding of the ETSA process beyond the interaction of single NPs during self-assembly and finds new trends for controlling the emulsion (i.e., the confinement) for an optimized large-scale SP synthesis.

\section{ASSOCIATED CONTENT}

\section{Supporting Information}

The Supporting Information is available free of charge at https://pubs.acs.org/doi/10.1021/acs.jpcb.0c07306.

Starting material and sample evaluation, overview micrographs showing example outcomes, DoE design, model building and confirmation, and optimal formulations for dense and microporous SPs (PDF).

\section{AUTHOR INFORMATION}

\section{Corresponding Author}

Berta Domènech - Institute of Advanced Ceramics, Hamburg University of Technology, Hamburg 21073, Germany; (i) orcid.org/0000-0003-2042-4428; Email: berta.domenech@tuhh.de

\section{Authors}

Alexander Plunkett - Institute of Advanced Ceramics, Hamburg University of Technology, Hamburg 21073, Germany; (i) orcid.org/0000-0002-2356-113X

Catriona Eldridge - Department of Materials Science and Metallurgy, University of Cambridge, Cambridge CB3 OFS, U.K. 
Gerold A. Schneider - Institute of Advanced Ceramics, Hamburg University of Technology, Hamburg 21073, Germany

Complete contact information is available at:

https://pubs.acs.org/10.1021/acs.jpcb.0c07306

\section{Notes}

The authors declare no competing financial interest.

\section{ACKNOWLEDGMENTS}

The authors gratefully acknowledge the financial support from the Deutsche Forschungsgemeinschaft (DFG, German Research Foundation, Project number 192346071, SFB 986). We further thank Dr. Kaline P. Furlan for the fruitful discussions and Tomasz Dobrowolski for his assistance in some of the laboratory work.

\section{REFERENCES}

(1) Boles, M. A.; Engel, M.; Talapin, D. V. Self-Assembly of Colloidal Nanocrystals: From Intricate Structures to Functional Materials. Chem. Rev. 2016, 116, 11220-11289.

(2) Begley, M. R.; Gianola, D. S.; Ray, T. R. Bridging functional nanocomposites to robust macroscale devices. Science 2019, 364, No. eaav4299.

(3) Xue, Z.; Yan, C.; Wang, T. From Atoms to Lives: The Evolution of Nanoparticle Assemblies. Adv. Funct. Mater. 2019, 29, 1807658.

(4) Wintzheimer, S.; Granath, T.; Oppmann, M.; Kister, T.; Thai, T.; Kraus, T.; Vogel, N.; Mandel, K. Supraparticles: Functionality from Uniform Structural Motifs. ACS Nano 2018, 12, 5093-5120.

(5) de Nijs, B.; Dussi, S.; Smallenburg, F.; Meeldijk, J. D.; Groenendijk, D. J.; Filion, L.; Imhof, A.; van Blaaderen, A.; Dijkstra, M. Entropy-driven formation of large icosahedral colloidal clusters by spherical confinement. Nat. Mater. 2015, 14, 56-60.

(6) Wang, D.; Hermes, M.; Kotni, R.; Wu, Y.; Tasios, N.; Liu, Y.; de Nijs, B.; van der Wee, E. B.; Murray, C. B.; Dijkstra, M.; et al. Interplay between spherical confinement and particle shape on the self-assembly of rounded cubes. Nat. Commun. 2018, 9, 2228.

(7) Wang, J.; Mbah, C. F.; Przybilla, T.; Englisch, S.; Spiecker, E.; Engel, M.; Vogel, N. Free Energy Landscape of Colloidal Clusters in Spherical Confinement. ACS Nano 2019, 13, 9005-9015.

(8) Wang, J.; Sultan, U.; Goerlitzer, E. S. A.; Mbah, C. F.; Engel, M.; Vogel, N. Structural Color of Colloidal Clusters as a Tool to Investigate Structure and Dynamics. Adv. Funct. Mater. 2019, 30, 1907730.

(9) Lacava, J.; Born, P.; Kraus, T. Nanoparticle clusters with Lennard-Jones geometries. Nano Lett. 2012, 12, 3279-3282.

(10) Marino, E.; Kodger, T. E.; Wegdam, G. H.; Schall, P. Revealing Driving Forces in Quantum Dot Supercrystal Assembly. Adv. Mater. 2018, 30, 1803433.

(11) Zhao, Y.; Shang, L.; Cheng, Y.; Gu, Z. Spherical colloidal photonic crystals. Accounts Chem. Res. 2014, 47, 3632-3642.

(12) Kim, S.-H.; Lee, S. Y.; Yi, G.-R.; Pine, D. J.; Yang, S.-M. Microwave-assisted self-organization of colloidal particles in confining aqueous droplets. J. Am. Chem. Soc. 2006, 128, 10897-10904.

(13) Park, J.-G.; Kim, S.-H.; Magkiriadou, S.; Choi, T. M.; Kim, Y.S.; Manoharan, V. N. Full-spectrum photonic pigments with noniridescent structural colors through colloidal assembly. Angew. Chem., Int. Ed. Engl. 2014, 53, 2899-2903.

(14) Vogel, N.; Utech, S.; England, G. T.; Shirman, T.; Phillips, K. R.; Koay, N.; Burgess, I. B.; Kolle, M.; Weitz, D. A.; Aizenberg, J. Color from hierarchy: Diverse optical properties of micron-sized spherical colloidal assemblies. Proc. Natl. Acad. Sci. U.S.A. 2015, 112, $10845-10850$.

(15) Xie, Z.; Cao, K.; Zhao, Y.; Bai, L.; Gu, H.; Xu, H.; Gu, Z.-Z. An optical nose chip based on mesoporous colloidal photonic crystal beads. Adv. Mater. 2014, 26, 2413-2418.
(16) Egly, S.; Fröhlich, C.; Vogel, S.; Gruenewald, A.; Wang, J.; Detsch, R.; Boccaccini, A. R.; Vogel, N. Bottom-Up Assembly of Silica and Bioactive Glass Supraparticles with Tunable Hierarchical Porosity. Langmuir 2018, 34, 2063-2072.

(17) Furlan, K. P.; Larsson, E.; Diaz, A.; Holler, M.; Krekeler, T.; Ritter, M.; Petrov, A. Y.; Eich, M.; Blick, R.; Schneider, G. A.; et al. Photonic materials for high-temperature applications: Synthesis and characterization by X-ray ptychographic tomography. Appl. Mater. Today 2018, 13, 359-369.

(18) do Rosário, J. J.; Häntsch, Y.; Pasquarelli, R. M.; Dyachenko, P. N.; Vriend, E.; Petrov, A. Y.; Furlan, K. P.; Eich, M.; Schneider, G. A. Advancing the fabrication of YSZ-inverse photonic glasses for broadband omnidirectional reflector films. J. Eur. Ceram. Soc. 2019, 39, 3353-3363.

(19) Shang, G.; Häntsch, Y.; Furlan, K. P.; Janßen, R.; Schneider, G. A.; Petrov, A.; Eich, M. Highly selective photonic glass filter for saturated blue structural color. APL Photonics 2019, 4, 046101.

(20) Häntsch, Y.; Shang, G.; Petrov, A.; Eich, M.; Schneider, G. A. YSZ Hollow Sphere Photonic Glasses: Tailoring Optical Properties for Highly Saturated Non-Iridescent Structural Coloration. Adv. Opt. Mater. 2019, 7, 1900428.

(21) Brandt, K.; Wolff, M. F. H.; Salikov, V.; Heinrich, S.; Schneider, G. A. A novel method for a multi-level hierarchical composite with brick-and-mortar structure. Sci. Rep. 2013, 3, 2322.

(22) Dreyer, A.; Feld, A.; Kornowski, A.; Yilmaz, E. D.; Noei, H.; Meyer, A.; Krekeler, T.; Jiao, C.; Stierle, A.; Abetz, V.; et al. Organically linked iron oxide nanoparticle supercrystals with exceptional isotropic mechanical properties. Nat. Mater. 2016, 15, $522-528$

(23) Domènech, B.; Tan, A. T. L.; Jelitto, H.; Zegarra Berodt, E.; Blankenburg, M.; Focke, O.; Cann, J.; Cem Tasan, C.; Colombi Ciacchi, L.; Müller, M.; et al. Strong Macroscale Supercrystalline Structures by 3D Printing Combined with Self-Assembly of Ceramic Functionalized Nanoparticles. Adv. Eng. Mater. 2020, 22, 2000352.

(24) Giuntini, D.; Torresani, E.; Chan, K. T.; Blankenburg, M.; Saviot, L.; Bor, B.; Domènech, B.; Shachar, M.; Müller, M.; Olevsky, E. A.; et al. Iron oxide-based nanostructured ceramics with tailored magnetic and mechanical properties: development of mechanically robust, bulk superparamagnetic materials. Nanoscale Adv. 2019, 1, 3139-3150.

(25) Bor, B.; Giuntini, D.; Domènech, B.; Swain, M. V.; Schneider, G. A. Nanoindentation-based study of the mechanical behavior of bulk supercrystalline ceramic-organic nanocomposites. J. Eur. Ceram. Soc. 2019, 39, 3247-3256.

(26) Domènech, B.; Kampferbeck, M.; Larsson, E.; Krekeler, T.; Bor, B.; Giuntini, D.; Blankenburg, M.; Ritter, M.; Müller, M.; Vossmeyer, T.; et al. Hierarchical supercrystalline nanocomposites through the self-assembly of organically-modified ceramic nanoparticles. Sci. Rep. 2019, 9, 3435.

(27) Domènech, B.; Plunkett, A.; Kampferbeck, M.; Blankenburg, M.; Bor, B.; Giuntini, D.; Krekeler, T.; Wagstaffe, M.; Noei, H.; Stierle, A.; et al. Modulating the Mechanical Properties of Supercrystalline Nanocomposite Materials via Solvent-Ligand Interactions. Langmuir 2019, 35, 13893-13903.

(28) Tadros, T. F. Emulsions: Formation, stability, industrial applications; De Gruyter graduate; De Gruyter: Berlin and Boston, 2016.

(29) Marino, E.; Keller, A. W.; An, D.; van Dongen, S.; Kodger, T. E.; MacArthur, K. E.; Heggen, M.; Kagan, C. R.; Murray, C. B.; Schall, P. Favoring the Growth of High-Quality, Three-Dimensional Supercrystals of Nanocrystals. J. Phys. Chem. C 2020, 124, 1125611264.

(30) Saldanha, P. L.; Lesnyak, V.; Manna, L. Large scale syntheses of colloidal nanomaterials. Nano Today 2017, 12, 46-63.

(31) Bai, F.; Wang, D.; Huo, Z.; Chen, W.; Liu, L.; Liang, X.; Chen, C.; Wang, X.; Peng, Q.; Li, Y. A versatile bottom-up assembly approach to colloidal spheres from nanocrystals. Angew. Chem., Int. Ed. Engl. 2007, 46, 6650-6653. 
(32) Duan, H.; Wang, D.; Kurth, D. G.; Möhwald, H. Directing self-assembly of nanoparticles at water/oil interfaces. Angew. Chem., Int. Ed. Engl. 2004, 43, 5639-5642.

(33) Lacava, J.; Ouali, A.-A.; Raillard, B.; Kraus, T. On the behaviour of nanoparticles in oil-in-water emulsions with different surfactants. Soft Matter 2014, 10, 1696-1704.

(34) Velev, O. D.; Furusawa, K.; Nagayama, K. Assembly of Latex Particles by Using Emulsion Droplets as Templates. 1. Microstructured Hollow Spheres. Langmuir 1996, 12, 2374-2384.

(35) Velev, O. D.; Furusawa, K.; Nagayama, K. Assembly of Latex Particles by Using Emulsion Droplets as Templates. 2. Ball-like and Composite Aggregates. Langmuir 1996, 12, 2385-2391.

(36) Isojima, T.; Suh, S. K.; Vander Sande, J. B.; Hatton, T. A. Controlled assembly of nanoparticle structures: spherical and toroidal superlattices and nanoparticle-coated polymeric beads. Langmuir 2009, 25, 8292-8298.

(37) Davies, J. T. A quantitative kinetic theory of emulsion type. I. Physical chemistry of the emulsifying agent. Proceedings of 2nd International Congress Surface Activity, 1957.

(38) Dean, A.; Voss, D.; Draguljić, D.; Dean, A. M. Design and analysis of experiments. Springer Texts in Statistics, 2nd ed.; Springer International Publishing and Springer: Cham, 2017.

(39) Murray, P. M.; Bellany, F.; Benhamou, L.; Bučar, D.-K.; Tabor, A. B.; Sheppard, T. D. The application of design of experiments (DoE) reaction optimisation and solvent selection in the development of new synthetic chemistry. Org. Biomol. Chem. 2016, 14, 2373-2384.

(40) Bowden, G. D.; Pichler, B. J.; Maurer, A. A Design of Experiments (DoE) Approach Accelerates the Optimization of Copper-Mediated 18F-Fluorination Reactions of Arylstannanes. Sci. Rep. 2019, 9, 11370.

(41) Sakia, R. M. The Box-Cox Transformation Technique: A Review. J. R. Stat. Soc. - Ser. D Statistician 1992, 41, 169-178.

(42) Israelachvili, J. The science and applications of emulsions-an overview. Colloids Surf., A 1994, 91, 1-8.

(43) Charcosset, C.; Limayem, I.; Fessi, H. The membrane emulsification process-a review. J. Chem. Technol. Biotechnol. 2004, 79, 209-218.

(44) Biswal, N. R.; Rangera, N.; Singh, J. K. Effect of Different Surfactants on the Interfacial Behavior of the n-Hexane-Water System in the Presence of Silica Nanoparticles. J. Phys. Chem. B 2016, 120, 7265-7274.

(45) Atanase, L.-I.; Lasuye, T.; Stasik, B.; Riess, G. Colloidal characteristis of vinyl alcohol-vinyl acetate copolymers by complex formation with sodium dodecyl sulphate. Rev. Roum. Chim. 2009, 54, $577-581$.

(46) Liu, W.; Kappl, M.; Butt, H.-J. Tuning the Porosity of Supraparticles. ACS Nano 2019, 13, 13949-13956.

(47) Matsumoto, S.; Makino, H.; Ueno, A. Formation of W/O/W Emulsions through the Simple Agitation of Oil-Water Mixtures. J. Jpn. Oil Chem. Soc. 1987, 36, 320-327. 